\title{
Influence of Gap Junction Dynamics on the Stability of Reentrant Waves in Cardiac Tissue
}

\author{
Claudia Hawks ${ }^{1}$, Jorge Elorza ${ }^{1}$, Blas Echebarria ${ }^{2}$, \\ Inma R. Cantalapiedra ${ }^{2}$, Angelina Peñaranda ${ }^{2}$, Jean Bragard ${ }^{1}$ \\ ${ }^{1}$ Dept. of Physics and Applied Math., University of Navarra, 31008 Pamplona, Spain \\ ${ }^{2}$ Dept. of Applied Physics, Polytechnic University of Catalonia, 08028 Barcelona, Spain
}

\begin{abstract}
Constant conductances are often assumed when modeling cardiac tissue. However experimental evidences have shown that gap junctions (GJ) actually connect adjacent cardiac myocytes. These GJ are complex proteins of the connexin family (Cx40; Cx43; Cx45 are the most common in human). These GJ modify the conductances between cardiac cell through a dynamical process. The aim of this study is to develop a bidomain model of the cardiac tissue where the dynamics of the connexins is also included. In particular we will compare the differences associated with the use of a monodomain versus bidomain formulation in inducing intra-cellular conductance variations. We have found that the monodomain formulation gives conductance variations a factor four to five larger with respect to the bidomain formulation.
\end{abstract}

\section{Introduction}

Gap junctions (GJ) are membrane channels that connect the cytoplasm of adjacent cells and allow for cell to cell current transfers. GJ are assembly of proteins and they are mainly three connexin types found in mammalian cardiac cells: Cx_40 ; Cx_43 and Cx_45 [1,2]. In the recent years a lot of attention has been drawn towards the role of GJ and connexin in the possible generation of arrhythmias. In the present work we propose to study the influence of the GJ dynamics on the conductance variations of the cardiac tissue. We will use two different types of cardiac tissue formulations that are: bidomain and monodomain.

\section{Models}

There are two concurrent models to describe the spatial electrical activity of the heart that are the so-called monodomain and bidomain models. The latter formulation offers a better description of the cardiac tissue because it includes both fundamental fields i.e. the intracellular elec- trical potential $\phi_{i}$ and the extracellular electrical potential $\phi_{e}$. On the other side, the monodomain formulation combines the two previous fields to write the transmembrane potential $V_{m}=\phi_{i}-\phi_{e}$ and the spatial description of the electrical activity is written in term of this single transmembrane potential. Furthermore $V_{m}$ is the quantity most easily accessible for direct experimental measurement in cardiac tissue. Except in the cases when one studies defibrillation and/or gap junction dynamics it can be shown that there exists an equivalent formulation of the bidomain equations using the monodomain formulation as we will detail in Sec. 2.2. Let us start explaining the most general model that includes the Gap Junction dynamics.

\subsection{Bidomain Model}

In this section, we will detail the equations for the bidomain model that include also the GJ dynamics. The complete mathematical expressions for the model are as follows [3] :

$$
\begin{aligned}
& \frac{\partial s}{\partial t}=f\left(V_{m}, s\right) \\
& \frac{\partial V_{m}}{\partial t}+\frac{I_{m}}{\mathcal{C}}=\nabla \cdot\left(D_{i} \nabla V_{m}\right)+\nabla \cdot\left(D_{i} \nabla \phi_{e}\right) \\
& \nabla \cdot\left(\left(D_{i}+D_{e}\right) \nabla \phi_{e}\right)=-\nabla \cdot\left(D_{i} \nabla V_{m}\right)-\frac{I_{e x t}}{\chi \mathcal{C}}(3)
\end{aligned}
$$

where $\mathcal{C}$ is the membrane capacitance $\left(\approx 1 \mu \mathrm{F} / \mathrm{cm}^{2}\right), \chi$ is the cell surface to volume ratio $\left(\approx 1,400 \mathrm{~cm}^{-1}\right)$ and $s$ is a state vector that contains all the dynamical variables involved in the local description of the ionic currents across the membrane, where all ion transport takes place. The mathematical description of the opening and closing of the ion channels is described by a set of differential equations (1). The time scales associated with these equations vary from $0.1 \mathrm{~ms}$ up to $1 \mathrm{~s}$ and higher. Furthermore, the vector function $f\left(V_{m}, s\right)$ in Eq. (1) is highly nonlinear. Consequently, the equations are stiff and are challenging to solve from the numerical point of view. Typical values for the 
conductivities [4] are $D_{i}=1.510^{-3}$, and $D_{e}=1.510^{-3}$, all in units of $\mathrm{cm}^{2} / \mathrm{ms}$. Equation (3) is the Poisson equation (elliptic PDE) that relates the current in the intracellular region to the extracellular electrical potential $\phi_{e}$. Time does not appear explicitly in the Poisson equation and it must be solved simultaneously with Eq. (2) at every time step. The term $I_{\text {ext }}$ allows for the introduction of an external current as it happens during an external excitation of the cardiac tissue.

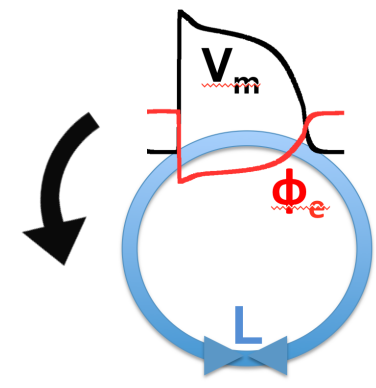

Figure 1. Schematic setup used in this study.

In the present study, we use a one dimensional geometry (annular ring) where the action potential wave is propagating. This is a simplified model for a two dimensional reentrant wave [5]. To solve Equations (1-3), we impose periodic boundary conditions on the ring.

\subsection{Monodomain Model}

The monodomain formulation can be obtained from the bidomain equations by setting $\phi_{e} \equiv 0$. By doing this we avoid solving the costly Poisson equation (3). In order to get the same wave speed for the action potential propagation it can be readily shown [3] that we need to rescale the conductivity as follows:

$$
D_{\text {mono. }}=\frac{D_{i} D_{e}}{D_{i}+D_{e}} .
$$

Therefore, in the comparison between monodomain and bidomain we will use here a nominal value for $D$ mono. $=$ $0.7510^{-3} \mathrm{~cm}^{2} / \mathrm{ms}$.

\subsection{Gap Junctions Dynamics}

The purposes of this work are twofold. First, is to build a full bidomain formulation that includes the GJ dynamics and then, in a second stage, compare the simulations on the annular ring obtained with the monodomain and bidomain models to evaluate the amplitude of changes due to each formulation. The dynamics of the gap junctions is modeled following the works of Lin et al. [1] and Desplantez et $a l$. [2]. When we discretize space, the index number $j$ corresponds to the cell number $j$. The gap junction between cell number $j-1$ and cell number $j$ has index number $j$ (following our convention) and its temporal dynamics is governed by the following differential equation:

$$
\frac{d g_{j}}{d t}=\frac{g_{j, s s}\left(\Delta \phi_{i}\right)-g_{j}}{\tau_{g}\left(\Delta \phi_{i}\right)},
$$

where $\Delta \phi_{i}=\phi_{i}(j)-\phi_{i}(j-1)$ is the difference in intracellular electrical potential between the two adjacent cells of the $g_{j}$. The steady state value in Eq.(5) depends on the local instantaneous $\Delta \phi_{i}$ following this equation:

$$
g_{j, s s}=\frac{g_{j, \max }-g_{j, \min }}{1+\exp \left[A\left(\Delta \phi_{i}-V_{1 / 2}\right)\right]}+g_{j, \min },
$$

and the time scale in Eq.(5) is given by $\tau_{g}=$ $A_{\tau} \exp \left[-B_{\tau}\left|\Delta \phi_{i}\right|\right]$. Once the gap junction values are computed, we use them in Eqs. $(2,3)$ through the relation:

$$
D_{i}\left(x_{j}, t\right)=\bar{D}_{i} g_{j}(t),
$$

where $\bar{D}_{i}$ is the constant nominal value for the intracellular conductivity $\bar{D}_{i}=1.510^{-3} \mathrm{~cm}^{2} / \mathrm{ms}$. The parameter values entering Eqs. $(5,6)$ are taken from the works of Lin and Desplantez [1,2] and they are gathered in Table (1) for completion. We also have the following relations for the parameters: $A=z / 26.714(\mathrm{mV})^{-1} ; A_{\tau}=109,900$ $(\mathrm{ms}) ; B_{\tau}=1 / 11.8(\mathrm{mV})^{-1} ; g_{j, \max }\left(\Delta \phi_{i}=0\right)=1$. The dependance of $g_{j, s s}$ as a function of $\Delta \phi_{i}$ is displayed in Fig. 2.

Table 1. Values for the Gap Junction dynamics. Here below, the parameter values are for $\left(\Delta \phi_{i}<0 / \Delta \phi_{i} \geq 0\right)$

\begin{tabular}{lccc}
\hline \hline Connexin type & $V_{1 / 2}(\mathrm{mV})$ & $g_{j, \min }$ & $z$ \\
\hline $\mathrm{Cx}_{43-43}$ & $-60.8 / 62.9$ & $0.26 / 0.25$ & $-3.4 / 2.9$ \\
$\mathrm{Cx}_{43-45}$ & $-11.7 / 134.4$ & $0.05 / 0.05$ & $-2.1 / 0.6$ \\
$\mathrm{Cx}_{45-43}$ & $134.4 /-11.7$ & $0.05 / 0.05$ & $0.6 /-2.1$ \\
\hline \hline
\end{tabular}

\subsection{Membrane Models}

From a dynamical point of view, the heart is an excitable medium. This means that a perturbation that overcomes a certain threshold produces an action potential (nonlinear response) that propagates as an electric wave throughout the entire domain with a characteristic shape (measured by the action potential duration APD and the diastolic interval DI) and the wave speed.

Since the 1960s, many differential-equations-based models to describe the kinetics of the cell membrane have been developed. In some cases, models have evolved to become quite complex by representing cellular processes in detail. Other models, e.g. the three-variable Fenton and Karma model [6], represent quite faithfully the propagation of the action potential without the numerical burden 


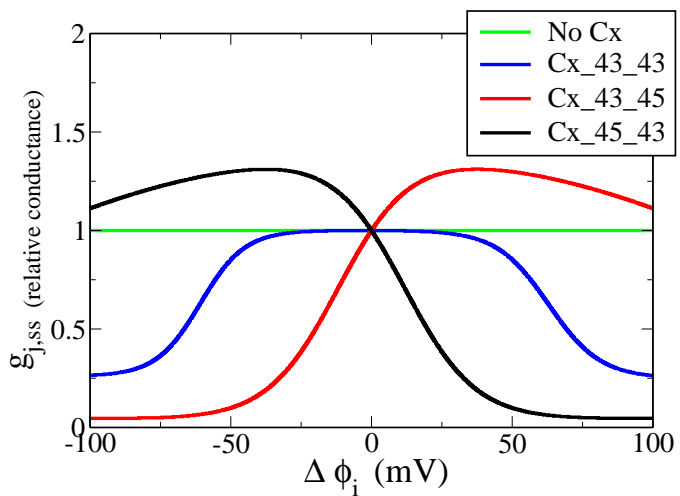

Figure 2. Steady state values of the gap junction $g_{j, s s}$ as a function of $\Delta \phi_{i}$.

associated with the large numbers of variables in the more complex models. In 2010, Cantalapiedra et al. [7,8] developed a five-variable model containing a specific formulation for the transient outward $K^{+}$current, which is important in describing action potentials associated with the Brugada syndrome. In this work, we use the Cantalapiedra et al. model $[7,8]$ with the model parameter fitted to describe human ventricular myocytes.

\subsection{Computation}

To solve Eqs. (1-3) numerically, it is necessary to discretize space and time. For the spatial discretization, the finite-volume method is used [3]. This method is preferred because it conserves exactly the charges moving from a reference volume to the next. The time discretization uses a simple forward-Euler method. The most costly part of the computation comes from the Poisson equation Eq. (3), which we solve using the PETSc package [9]. The spatial discretization of the equation leads to a system of the form $A x=b$, where $A$ is a large sparse positive semi-definite matrix and the vector $x$ contains the unknowns $\phi_{e}$. Due to the fact that we deal with GJ dynamics this equation has a matrix $A$ that is time dependent. This means that we need to redefine the matrix $A$ for the PETSc solver at each time step and this leads to double the CPU time with respect to the constant $A$ matrix case. Here we have choosen $\Delta x=0.01 \mathrm{~cm}$ and $\delta t=0.01 \mathrm{~ms}$, which leads to a very good accuracy. Note also that the monodomain calculations are one order of magnitude faster than the bidomain.

\section{Results}

The main objective of this study is to compare the effect of the gap junction dynamics (GJ) in the bidomain and monodomain formulation. We start the simulations with a

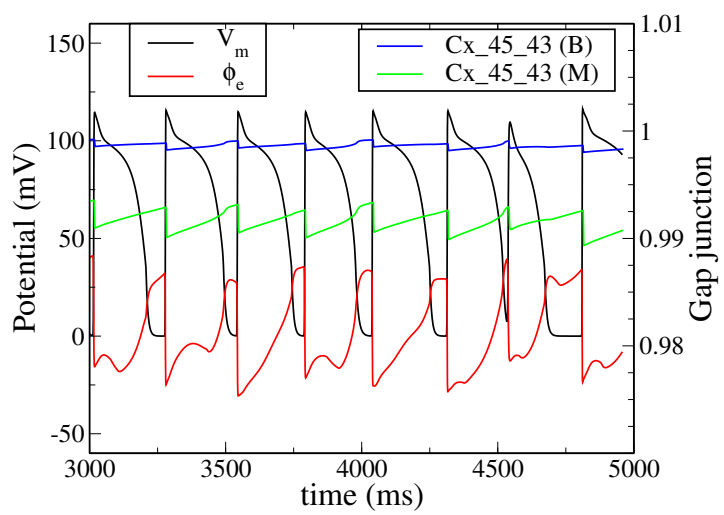

Figure 3. Time evolution of the membrane potential $V_{m}$ and the extracellular potential $\left(\phi_{e}\right)$ measured at a specific location on the ring $L / 4$. The evolution of the GJ is also shown for the Cx_45_43 connexin in a model of monodomain and bidomain. Here, the system size is $L=8.5$ $\mathrm{cm}$. Note that the scale for the GJ is on the RHS of the graph and the scale for $V_{m}$ and $\phi_{e}$ is located on the LHS of the graph and are given in dimensional units $(\mathrm{mV})$.

single wave propagating along the one dimensional ring as schematically shown in Fig. 1. After the system has established to a steady state (for large system size $L>9.6$ $\mathrm{cm}$ ) or that some transient has elapsed (for small system size $L \leq 9.6 \mathrm{~cm}$ ) we make measurements on the wave speed; the APD; the DI; and the gap junction dynamics. After the measurements are performed (on 20 revolutions of the wave on the ring), we reduce the system size (circumference) and repeat the process. We observe that for all connexin types that we have studied and for both formulations (i.e. monodomain and bidomain), there are few differences on the wave characteristics (speed, APD and DI).

Typical temporal dynamics of $V_{m}, \phi_{e}$ and $g_{j}$ measured at a specific location on the ring $(L / 4)$ are shown in Fig. 3. In this case the system size is $L=8.5 \mathrm{~cm}$ and the dynamics on the ring corresponds to discordant alternans waves. One observes that the GJ dynamics is mostly affected during the depolarization phase of the action potential as expected. Furthermore we observe that the scale of variation of the GJ dynamics is about a factor four to five greater when considering a monodomain rather than bidomain formulation. This can be readily explained by the fact that the $\phi_{i}$ variation from cell to cell (used in the the bidomain formulation) is much smaller than the $V_{m}$ variation form cell to cell used in the monodomain variation.

In Fig. 4 we have displayed the wave speed as a function of the ring size (circumference) for the different type of connexins and also for the case without connexins (constant conductivity). Fig. 4 has been drawn using the monodomain formulation, the wave speed is very similar (in- 
distinguishable) when using the bidomain formulation (not shown in Fig. 4). One clearly sees in the inset of Fig. 4 the transition to a discordant-alternans dynamics when the system size is reduced below $L<9.6 \mathrm{~cm}$.

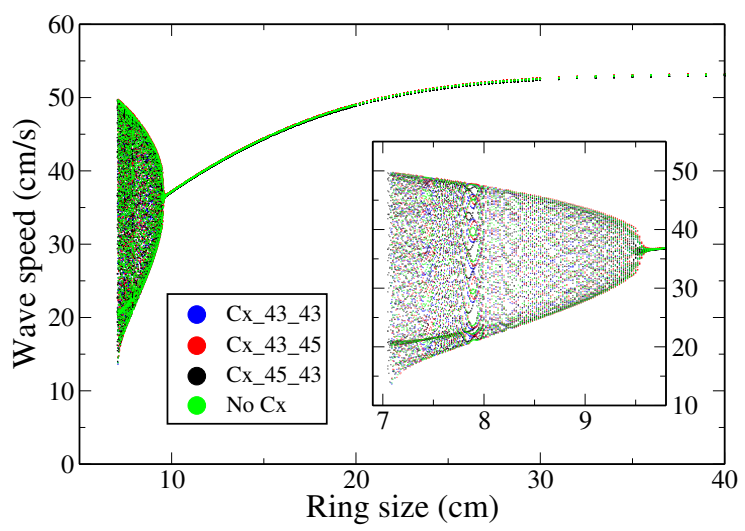

Figure 4. Variation of the wave speed as function of the ring size (circumference). The inset shows the discordantalternans region.

Figure 5 shows the spatial average of the gap junction $<g_{j}>$ as a function of the system size. The upper (lower) plot corresponds to the bidomain (monodomain) formulation, respectively. The LHS scale corresponds to connexin types Cx_43_45 and Cx_45_43 while the right scale corresponds to the $\mathrm{Cx} \_43 \_43$ connexin (here we report $1-<g_{j}>$ because of the relative small variations induced by this connexin).

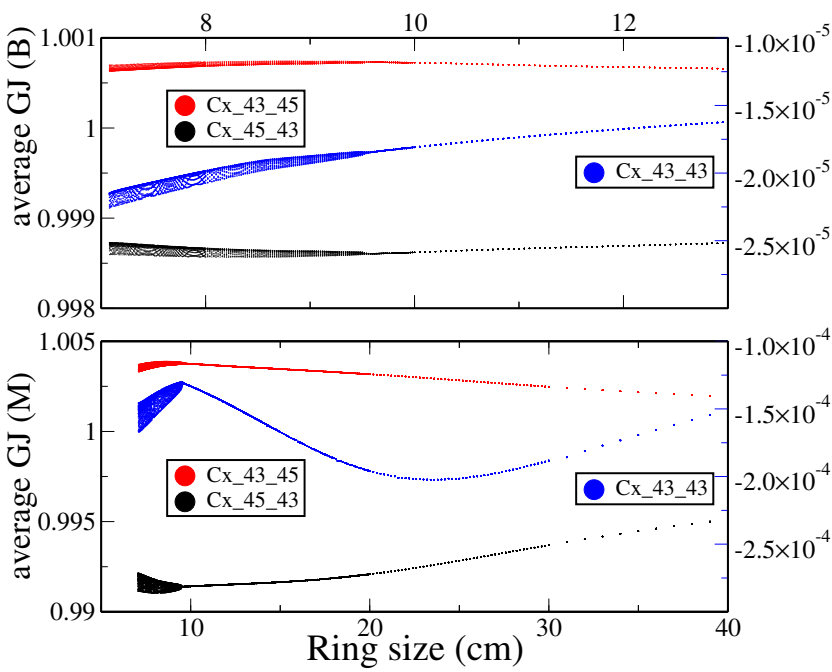

Figure 5. $\left\langle g_{j}>\right.$ for different connexin types as a function of the system size. For connexin Cx_43_43, $1-<$ $g_{j}>$ are reported on the right $y-$ axes. Upper (lower) plot correspond to bidomain (monodomain) formulation.

\section{Conclusions}

We have compared the influence of the connexin types (Cx_43_43; Cx_43_45 and Cx_45_43) in modifying the conductivity of the system when considering either the monodomain or bidomain formulation. In normal conditions, the influence is very small and comparable to the case without GJ. The variations induced in the monodomain formulation appear to be four to five times larger than in a bidomain formulation while still very close to one. The next step in our study is to artificially induce "pathological" conditions in the tissue by lowering the GJ conductances and modifying the parameters associated to the GJ dynamics to see the extend of the effect on the characteristics of the wave propagation (wave speed; APD and DI). We expect that these modifications will induce larger variations in the wave characteristics that may lead to important pro-arrhythmic effects.

\section{Acknowledgements}

Financial support from the Spanish Ministry of Economy is acknowledged through project number FIS201128820. C.H. acknowledges ADA support for a PhD grant.

\section{References}

[1] Lin X, Crye M, Veenstra R. Regulation of Connexin 43 Gap Junctional Conductance by Ventricular Action Potential. Circ Res 2003;93:63-73.

[2] Desplantez T, Halliday D, Dupont E, Weingart R. Cardiac connexins $\mathrm{Cx} 43$ and $\mathrm{Cx} 45$ : formation of diverse gap junction channels with diverse electrical properties. Eur J Physiol 2004;448:363-375.

[3] Sachse F. Computational Cardiology. Springer, BerlinHeidelberg, 2004.

[4] Aguel F, Eason J, Trayanova N. Advances in modeling cardiac defibrillation. IJBC 2003;13(12):3791-3803.

[5] Glass L, Josephson ME. Resetting and Annihilation of Reentrant Abnormally Rapid Heartbeat. Physical Review Letters 1995;75(10):2059-2062.

[6] Fenton F, Karma A. Vortex dynamics in three-dimensional continuous myocardium with fiber rotation: Filament instability and fibrillation. Chaos 1998;8(1):20-47.

[7] Cantalapiedra I, Peñaranda A, Echebarria B, Bragard J. Phase-2 reentry in cardiac tissue: Role of the slow calcium pulse. Phys Rev E 2010;82(1)):1907.

[8] Peñaranda A, Cantalapiedra I, Bragard J, Echebarria B. Cardiac dynamics: A simplified model for action potential propagation. Theo Biol and Med Modelling 2012;9:50.

[9] Balay S, Buschelman K, Eijkhout V, Gropp W, Kaushik D, Knepley M, McInnes L, Smith B, Zhang H. In PETSc Users Manual. Argonne National Laboratory; 2008. 